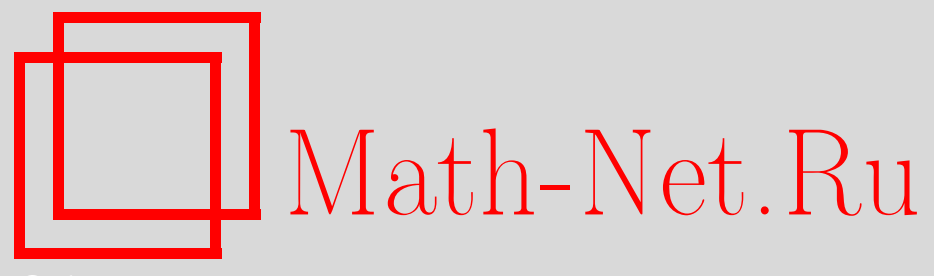

С. А. Гриценко, Задача Хуа Ло-кена с простыми числами, представимыми заданными квадратичными формами, Изв. РАН. Сер. матем., 2007, том 71, выпуск 3, 4560

DOI: https://doi.org/10.4213/im1137

Использование Общероссийского математического портала Math-Net.Ru подразумевает, что вы прочитали и согласны с пользовательским соглашением http://www.mathnet.ru/rus/agreement

Параметры загрузки:

IP : 52.205 .19 .152

26 апреля 2023 г., 13:26:12

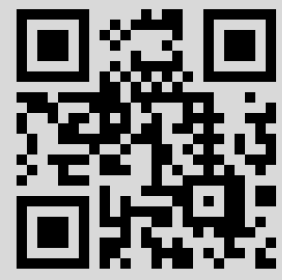




\title{
С. А. Гриценко \\ Задача Хуа Ло-кена с простыми числами, представимыми заданными квадратичными формами
}

\begin{abstract}
Решена задача Хуа Ло-кена с простыми числами, представимыми заданными примитивными положительно определенными бинарными квадратичными формами, дискриминанты которых совпадают с дискриминантами мнимых квадратичных полей, в которых квадратичные формы распадаются на линейные множители.

Библиография: 10 наименований.
\end{abstract}

\section{Введение}

В монографии [1] излагается теория проблемы Варинга-Гольдбаха, в частности выводится асимптотическая формула для числа решений уравнения

$$
p_{1}^{2}+p_{2}^{2}+p_{3}^{2}+p_{4}^{2}+p_{5}^{2}=N
$$

в простых числах $p_{1}, \ldots, p_{5}$.

Задачу изучения уравнения (1) будем называть задачей Хуа Ло-кена.

В настоящей статье выводится асимптотическая формула для числа решений уравнения (1) в простых числах $p_{1}, \ldots, p_{5}$, на которые наложены ограничения, состоящие в том, что каждое простое число $p_{j}, j=1, \ldots, 5$, представляется некоторой заданной бинарной квадратичной формой.

Заметим, что если эти квадратичные формы являются одноклассными, то задача сводится по существу к решению задачи Хуа Ло-кена с простыми числами, лежащими в определенных арифметических прогрессиях. В случае, когда разности этих арифметических прогрессий являются константами или растут с ростом $N$ не слишком быстро, решение такой задачи мало чем отличается от решения классической задачи Хуа Ло-кена. То же относится и к задаче получения асимптотической формулы для числа решений уравнения (1) в простых числах, представимых всеми квадратичными формами заданных дискриминантов.

В случае, если квадратичные формы, представляющие простые числа, являются многоклассными, вопрос о представимости натуральных чисел такими формами не может быть сведен к вопросу о попадании этих чисел в какие-то классы вычетов по какому-либо модулю (в связи с этим см. [2, с. 271]).

Поэтому для решения задачи, помимо информации о распределении простых чисел, представимых квадратичными формами, в арифметических прогрессиях требуются оценки некоторых специальных тригонометрических сумм.

Работа выполнена при поддержке Министерства образования и науки РФ в рамках программы "Развитие научного потенциала высшей школы" (грант РНП.2.1.1.3263) и Белгородского государственного университета (грант ВГК 007-04). 
Далее под квадратичными формами мы будем понимать примитивные положительно определенные бинарные квадратичные формы, дискриминанты которых совпадают с дискриминантами мнимых квадратичных полей, в которых эти формы разлагаются на линейные множители.

Дискриминанты квадратичных форм везде предполагаются константами, хотя из доказательств основной теоремы и лемм видно, что имеется возможность некоторого их роста вместе с ростом параметра $N$.

В дальнейшем будут использоваться следующие обозначения: $p, p_{1}, p_{2}, \ldots-$ простые числа; $\mathcal{K}$ - мнимое квадратичное поле с дискриминантом $\delta_{\mathcal{K}} ; D=\left|\delta_{\mathcal{K}}\right|$; $A, B, C$ - целые идеалы $\mathbb{Z}_{\mathcal{K}} ; P$ - простые идеалы $\mathbb{Z}_{\mathcal{K}} ; N(A)$ - норма целого идеала $A$ кольца $\mathbb{Z}_{\mathcal{K}} ; \mathcal{A}, \mathcal{B}, \mathcal{C}$ - классы идеалов $\mathbb{Z}_{\mathcal{K}} ;$

$$
\begin{gathered}
\chi(q ; k, l)= \begin{cases}1, & \text { если } q \equiv l(\bmod k), \\
0 & \text { в противном случае; }\end{cases} \\
S(m, q)=\sum_{l=1}^{q} \exp \left\{2 \pi i \frac{m l^{2}}{q}\right\},
\end{gathered}
$$

- суммы Гаусса; $\sum_{a=0}^{* b-1}$ обозначает суммирование по $a$, взаимно простым с $b$, в указанных пределах;

$$
\begin{aligned}
& S^{\prime}(a, q)=\sum_{l=1}^{q} \exp \left\{2 \pi i \frac{a}{q} l^{2}\right\} \\
& S_{j}^{\prime \prime}(a, q)=\sum_{l=1}^{q} \chi_{j}(l) \exp \left\{2 \pi i \frac{a}{q} l^{2}\right\},
\end{aligned}
$$

где $\chi_{j}(l), j=1, \ldots, 5,-$ характеры квадратичных полей, в которых квадратичные формы $Q_{j}$ разлагаются на линейные множители; $h_{j}$ - число классов эквивалентности квадратичных форм того же дискриминанта, что форма $Q_{j}$, $j=1, \ldots, 5$

$$
S_{Q}(\alpha)=\sum_{p \leqslant \sqrt{N}}^{\prime} e^{2 \pi i \alpha p^{2}}
$$

где штрих означает, что суммирование идет по простым числам, представимым квадратичной формой $Q ; \pi_{\mathcal{C}}(x, q, l, \mathcal{C})$ - число простых идеалов $\mathbb{Z}_{\mathcal{K}}$ из класса $\mathcal{C}$, нормы которых не превосходят $x$ и сравнимы с $l$ по модулю $q ; \pi_{Q}(x, q, l)$ - число простых чисел, не превосходящих $x$, представимых квадратичной формой $Q$ и сравнимых с $l$ по модулю $q$.

\section{$\S 1$. Лемма о квадратичных формах и формулировка основного результата}

Лемма 1. Пусть $Q(x, y)$ - бинарная положительно определенная примитивная квадратичная форма, разлагающаяся в поле $\mathcal{K}=\mathbb{Q}(\sqrt{d})$ на линейнъе множители. Тогда в кольце иельх алгебраических чисел поля $\mathbb{Q}(\sqrt{d})$ существует целый идеал $A$ с иелым базисом $\omega_{1}, \omega_{2}$ такой, что $Q(x, y)=$ $\frac{N\left(x \omega_{1}+y \omega_{2}\right)}{N(A)}$. Квадратичные формы эквивалентны тогда и только тогда, когда соответствующие им идеалы с указанным свойством эквивалентны. 
ДокАЗАТЕЛЬСтво см. в [2, с. 160], [3, с. 215-216].

СледСтвиЕ 1. Пусть выполнены условия леммы 1 и идеал А принадлежит классу идеалов $\mathcal{A}$. Тогда квадратичная форма $Q(x, y)$ представляет те и только те простые числа р, которые являются нормами простых идеалов из класca $\mathcal{A}^{-1}$.

ДоказАтельство. Пусть $P \in \mathcal{A}^{-1}-$ простой идеал, $N(P)=p$. Тогда $P A=(\xi)$, где $\xi$ - число из идеала $A, \xi=x \omega_{1}+y \omega_{2}$.

Взяв норму от обеих частей равенства $P A=(\xi)$, получим

$$
Q(x, y)=\frac{N\left(x \omega_{1}+y \omega_{2}\right)}{N(A)}=p .
$$

Обратно, пусть $Q\left(x_{0}, y_{0}\right)=p, \xi_{0}=x_{0} \omega_{1}+y_{0} \omega_{2}$. Разделим идеал $\left(\xi_{0}\right)$ на $A$ : $\left(\xi_{0}\right)=A B$, где $B$ - целый идеал.

Очевидно, $B \in \mathcal{A}^{-1}$. Кроме того, по предположению $N(B)=\frac{N\left(\xi_{0}\right)}{N(A)}=p$, следовательно, $B$ - простой идеал.

СлеДСтвиЕ 2. Пусть выполнены условия леммы 1, форме $Q$ соответствует идеал $A$ из класса $\mathcal{A}, l$ и q-целые взаимно простые числа. Пусть

$$
\alpha(Q)=\left\{\begin{array}{lll}
2, & \text { если } & \mathcal{A}=\mathcal{A}^{-1} \\
1, & \text { если } & \mathcal{A} \neq \mathcal{A}^{-1}
\end{array}\right.
$$

Тогда

$$
\pi_{Q}(x, q, l)=\alpha(Q)^{-1} \pi\left(x, q, l, \mathcal{A}^{-1}\right)+O(\sqrt{x}) .
$$

ДокАЗАТЕЛьСтво. Пусть $p$ - простое число, $p \nmid \delta_{\mathcal{K}}$ и $p$ представляется формой $Q$. Докажем, что $\alpha(Q)$ равно числу простых идеалов из класса $\mathcal{A}^{-1}$ с нормами $p$.

Хорошо известно (см., например, [4, с. 232]), что $(p)$ представляется в виде произведения простых идеалов $(p)=P P_{1}, P \neq P_{1}, \quad N(P)=p, \quad N\left(P_{1}\right)=p$, причем такое представление единственно, и других идеалов с нормой $p$ нет.

В силу следствия 1 по крайней мере один из идеалов $P, P_{1}$ (например, $P$ ) принадлежит $\mathcal{A}^{-1}$. Тогда, очевидно, $P_{1} \in \mathcal{A}$.

Если $\mathcal{A} \neq \mathcal{A}^{-1}$, то $P_{1} \notin \mathcal{A}^{-1}$ и существует ровно один простой идеал из $\mathcal{A}^{-1}$ с нормой $p$.

Если же $\mathcal{A}=\mathcal{A}^{-1}$, то классу $\mathcal{A}^{-1}$ принадлежат ровно два простых идеала с нормой $p$.

Теперь наше утверждение следует из того, что в классе $\mathcal{A}^{-1}$ не более $\sqrt{x}$ простых идеалов $P$ таких, что $N(P)=p^{2}, p^{2} \leqslant x$, а число простых чисел, делящих $\delta_{\mathcal{K}}$, конечно.

Сформулируем основной результат статьи.

Теорема 1. Пусть $N \equiv 5(\bmod 24)$, и пусть $b>0-$ произвольная постоянная. Тогда для числа $I(N)$ решений уравнения

$$
p_{1}^{2}+p_{2}^{2}+p_{3}^{2}+p_{4}^{2}+p_{5}^{2}=N
$$


в простых числах $p_{1}, \ldots, p_{5}$, каждое из которых представимо заданной бинарной квадратичной формой $Q_{j}(x, y), j=1, \ldots, 5$, справедлива формула

$$
I(N)=\frac{1}{h_{1} \ldots h_{5} \alpha\left(Q_{1}\right) \ldots \alpha\left(Q_{5}\right)} \sigma\left(N, D_{1}, \ldots, D_{5}\right) H(N)+O\left(N^{\frac{3}{2}} \ln ^{-b} N\right),
$$

где

$$
\begin{gathered}
H(N)=\int_{-\infty}^{+\infty} J^{5}(z) e^{-2 \pi i z N} d z, \quad J(z)=\int_{2}^{\sqrt{N}} \frac{e^{-2 \pi i z x^{2}}}{\ln x} d x, \\
\sigma\left(N, D_{1}, \ldots, D_{5}\right)=\sum_{q=1}^{\infty} \gamma\left(q, N, D_{1}, \ldots, D_{5}\right), \\
\gamma\left(q, N, D_{1}, \ldots, D_{5}\right)=\sum_{a=0}^{q-1} \prod_{j=1}^{5}\left(\frac{S^{\prime}(a, q)}{\varphi(q)}+\frac{\chi\left(q ; D_{j}, 0\right) S_{j}^{\prime \prime}(a, q)}{\varphi(q)}\right) \exp \left\{-2 \pi i \frac{a}{q} N\right\}, \\
S^{\prime}(a, q)=\sum_{l=0}^{q-1} \exp \left\{2 \pi i \frac{a}{q} l^{2}\right\}, \quad S_{j}^{\prime \prime}(a, q)=\sum_{l=0}^{q-1} \chi_{j}^{*}(l) \exp \left\{2 \pi i \frac{a}{q} l^{2}\right\},
\end{gathered}
$$

$\chi_{j}(l), j=1, \ldots, 5,-$ характеры квадратичных полей, в которых квадратичные формы $Q_{j}$ разлагаются на линейные множители. При этом $H(N)=$ $\frac{\pi^{2}}{24} \frac{N^{\frac{3}{2}}}{\ln ^{5} \sqrt{N}}\left(1+O\left(\frac{\ln \ln N}{\ln N}\right)\right)$. Постоянная в знаке $O$ зависит лишь от $b$.

Доказательство проводится круговым методом. Кроме того, существенную роль играет функциональное уравнение ряда Дирихле специального вида из [5], на котором основана асимптотическая формула из [6].

Для доказательства теоремы 1 нам потребуются следующие леммы.

\section{§ 2. Леммы для вычисления интеграла по большим дугам}

Лемма 2. Справедливы следующие утверждения:

1) если $\left(q_{1}, q_{2}\right)=1$, то $S\left(a, q_{1} q_{2}\right)=S\left(a q_{2}, q_{1}\right) S\left(a q_{1}, q_{2}\right)$;

2) пусть $d=(m, q)$, тогда

$$
S(m, n, q)=\left\{\begin{array}{lll}
d S\left(\frac{m}{d}, \frac{n}{d}, \frac{q}{d}\right), & \text { если } & d \mid n, \\
0, & \text { если } & d \nmid n ;
\end{array}\right.
$$

3) если $(m, q)=1$, mo $|S(m, n, q)| \leqslant 2 \sqrt{q}$;

4) пусть $(q, 2)=1$, тогда

$$
S(1, q)=\left\{\begin{array}{lll}
\sqrt{q}, & \text { если } & q \equiv 1(\bmod 4), \\
i \sqrt{q}, & \text { если } & q \neq \equiv 1(\bmod 4)
\end{array}\right.
$$

5) пусть $(a, 2)=1$, тогда

$$
S\left(a, 2^{l}\right)=\left\{\begin{array}{lll}
0, & \text { если } & l=1, \\
2^{l / 2}\left(1+i^{a}\right), & \text { если } & l \text { четное }, \\
2^{(l+1) / 2} e^{\pi a / 4}, & \text { если } & l \text { нечетное. }
\end{array}\right.
$$


ДоказАТЕЛЬСтво см., например, в [7].

Лемма 3. Справедливы следующие утверждения:

1) если $\left(q_{1}, q_{2}\right)=1$, то $S^{\prime}\left(a, q_{1} q_{2}\right)=S^{\prime}\left(a q_{2}, q_{1}\right) S^{\prime}\left(a q_{1}, q_{2}\right)$;

2) пусть $p>2$ - простое число, $(a, p)=1$, тогда

$$
S^{\prime}\left(a, p^{l}\right)=\left\{\begin{array}{lll}
S(a, p)-1, & \text { если } \quad l=1 \\
0, & \text { если } \quad l>1
\end{array}\right.
$$

3) $\operatorname{nycms}(a, 2)=1$, тогда

$$
S^{\prime}\left(a, 2^{l}\right)=\left\{\begin{array}{lll}
-1, & \text { если } & l=1 \\
2 i^{a}, & \text { если } & l=2 \\
4 e^{\pi i / 4}, & \text { если } & l=3 \\
0, & \text { если } & l>3
\end{array}\right.
$$

Лемма 3 прямо следует из леммы 2.

Лемма 4. Пусть $(a, q)=1,(a, D k)=1, D-$ модуль характера х квадратичного поля, $\varepsilon>0$ - прочзвольно малое число. Справедливы оченки

$$
S^{\prime}(a, q) \ll q^{0.5+\varepsilon}, \quad S^{\prime \prime}(a, D k) \ll(D k)^{0.5+\varepsilon} .
$$

ДокАЗАТЕЛЬСтво. Из лемм 2 и 3 следует, что

$$
S^{\prime}(a, q) \ll \sqrt{q} \prod_{p \mid q} 2 \ll q^{0.5+\varepsilon} .
$$

Оценим $S^{\prime \prime}(a, D k)$. Пусть $k=q_{1} q_{2}$, где $\left(D, q_{2}\right)=1$, а $q_{1}-$ либо 1 , либо натуральное число, все простые делители которого делят $D$. Тогда

$$
\begin{aligned}
S^{\prime \prime}\left(a, D q_{1} q_{2}\right) & =\sum_{l=1}^{D q_{1} q_{2}} \chi(l) \exp \left\{2 \pi i \frac{a l^{2}}{D q_{1} q_{2}}\right\} \\
& =\chi\left(q_{2}\right) S^{\prime}\left(a D q_{1}, q_{2}\right) \sum_{l=1}^{D q_{1}} \chi(l) \exp \left\{2 \pi i \frac{a q_{2} l^{2}}{D q_{1}}\right\} .
\end{aligned}
$$

Пользуясь оценкой суммы $S^{\prime}\left(a D q_{1}, q_{2}\right)$, приходим к неравенству

$$
S^{\prime \prime}\left(a, D q_{1} q_{2}\right) \ll q_{2}^{0.5+\varepsilon}\left|\sum_{l=1}^{D q_{1}} \chi(l) \exp \left\{2 \pi i \frac{a q_{2} l^{2}}{D q_{1}}\right\}\right| .
$$

Далее,

$$
\sum_{l=1}^{D q_{1}} \chi(l) \exp \left\{2 \pi i \frac{a q_{2} l^{2}}{D q_{1}}\right\}=\sum_{l=1}^{D} \chi(l) \exp \left\{2 \pi i \frac{a q_{2} l^{2}}{D q_{1}}\right\} S\left(a q_{2} D, 2 a q_{2} l, q_{1}\right) .
$$

В силу леммы 2 имеем $S\left(a q_{2} D, 2 a q_{2} l, q_{1}\right)=0$, если $\left(D, q_{1}\right) \nmid 2 l$; в то же время $(D, l)=1$, поэтому без ограничения общности можно предположить, что $\left(D, q_{1}\right) \mid 2$, т. е. либо $q_{1}=1$, либо $q_{1}=2$. 
Пусть $D=2^{\alpha} D_{1}, \quad 0 \leqslant \alpha \leqslant 3, \quad D_{1}-$ нечетное бесквадратное число. Справедливо тождество

$$
\begin{aligned}
& \sum_{l=1}^{D q_{1}} \chi(l) \exp \left\{2 \pi i \frac{a q_{2} l^{2}}{D q_{1}}\right\} \\
& \quad=\sum_{l=1}^{2^{\alpha} q_{1}} \chi\left(l ; 2^{\alpha}\right) \exp \left\{2 \pi i \frac{r_{2} l^{2}}{2^{\alpha} q_{1}}\right\} \prod_{p \mid D_{1}} \sum_{l=1}^{p} \chi(l ; p) \exp \left\{2 \pi i \frac{r_{p} l^{2}}{p}\right\}
\end{aligned}
$$

где $\left(r_{2}, 2\right)=1, \quad\left(r_{p}, p\right)=1, \chi(l ; m)$ - характер $\chi$ по модулю $m$.

Таким образом, мы получили неравенство

$$
\left|\sum_{l=1}^{D q_{1}} \chi(l) \exp \left\{2 \pi i \frac{a q_{2} l^{2}}{D q_{1}}\right\}\right| \leqslant 16 \prod_{p \mid D_{1}}\left|S_{p}\right|
$$

где

$$
S_{p}=\sum_{l=1}^{p} \chi(l ; p) \exp \left\{2 \pi i \frac{r_{p} l^{2}}{p}\right\}
$$

Оценим $S_{p}$. Имеем

$$
\begin{aligned}
S_{p} & =\frac{1}{p-1} \sum_{y=1}^{p-1} \sum_{l=1}^{p} \chi(l y ; p) \exp \left\{2 \pi i \frac{r_{p} l^{2} y^{2}}{p}\right\} \\
\left|S_{p}\right|^{2} & \leqslant \frac{1}{p-1} \sum_{y=1}^{p-1}\left|\sum_{l=1}^{p} \chi(l ; p) \exp \left\{2 \pi i \frac{r_{p} l^{2} y^{2}}{p}\right\}\right|^{2} \\
& \leqslant \frac{2}{p-1} \sum_{y=1}^{p}\left|\sum_{l=1}^{p} \chi(l ; p) \exp \left\{2 \pi i \frac{r_{p} l^{2} y}{p}\right\}\right|^{2} \\
& \leqslant \frac{2}{p-1} \sum_{l_{1}=1}^{p-1} \sum_{l_{2}=1}^{p-1} \sum_{y=1}^{p} \exp \left\{2 \pi i \frac{r_{p}\left(l_{1}^{2}-l_{2}^{2}\right) y}{p}\right\} \leqslant 4 p
\end{aligned}
$$

следовательно, $\left|S_{p}\right| \leqslant 2 \sqrt{p}$.

Получено неравенство

$$
S^{\prime \prime}(a, D k) \ll 2^{\sigma} \sqrt{D k} q_{2}^{\varepsilon},
$$

где $\sigma$ - количество различных простых делителей числа $D_{1}$.

Пользуясь известным неравенством $2^{\sigma} \ll\left(D_{1}\right)^{\varepsilon}$, получаем требуемую оценку для $S^{\prime \prime}(a, D k)$.

Лемма 5. Пусть $l$ и $q$ - иелье взаимно простые числа, $1 \leqslant q \leqslant \ln ^{c_{1}} x$,

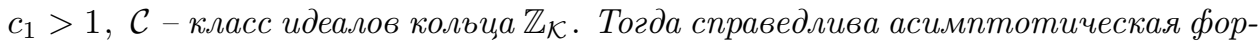
мула

$$
\pi_{\mathcal{C}}(x, q, l, \mathcal{C})=\frac{1+\chi(q ; D, 0) \chi_{1}(l)}{h \varphi(q)} \operatorname{Li} x+O\left(x \exp \left\{-c_{0}(\ln x)^{\frac{1}{20 c_{1}}}\right\}\right),
$$

где $c_{0}=c_{0}\left(c_{1}\right)>0$.

ДоказАтеЛьСтво см. в [6]. 


\section{§ 3. Леммы для оценки интеграла по малым дугам}

ОПРЕДЕЛЕНИЕ 1 . Пусть $A$ - идеал в $\mathbb{Z}_{\mathcal{K}}$, тогда функция $\mu_{\mathcal{K}}(A)$ определяется равенством

$$
\mu_{\mathcal{K}}(A)=\left\{\begin{array}{lll}
1, & \text { если } & A=\mathbb{Z}_{\mathcal{K}}, \\
(-1)^{r}, & \text { если } & A=P_{1} P_{2} \cdots P_{r}, \\
0, & \text { если } & \exists P, P^{2} \mid A .
\end{array}\right.
$$

ОПРЕДЕЛЕНИЕ 2. Пусть $A$ - идеал в $\mathbb{Z}_{\mathcal{K}}$, тогда функция $\Lambda_{\mathcal{K}}(N(A))$ определяется равенством

$$
\Lambda_{\mathcal{K}}(N(A))= \begin{cases}\ln N(P), & \text { если } A=P^{k}, k \geqslant 1, \\ 0 & \text { в противном случае. }\end{cases}
$$

Лемма 6. При $\operatorname{Re} s>1, u \geqslant 1$ справедливо тождество

$$
\begin{aligned}
\sum_{u<N(A)} & X(A) \Lambda_{\mathcal{K}}(N(A)) N^{-s}(A) \\
= & \sum_{N(C) \leqslant u} X(C) \mu_{\mathcal{K}}(C) N^{-s}(C) \sum_{A} X(A)(\ln N(A)) N^{-s}(A) \\
& -\sum_{N(C) \leqslant u} X(C) \mu_{\mathcal{K}}(C) N^{-s}(C) \\
& \times \sum_{N(B) \leqslant u} X(B) \Lambda_{\mathcal{K}}(N(B)) N^{-s}(B) \sum_{A} X(A) N^{-s}(A) \\
& -\sum_{u<N(A)} X(A) \Lambda_{\mathcal{K}}(N(A)) N^{-s}(A) \sum_{u<N(B)} X(B) \sum_{\substack{C \mid B \\
N(C) \leqslant u}} \mu_{\mathcal{K}}(C) N^{-s}(B) .
\end{aligned}
$$

ДоказАТЕльство. Пусть $\operatorname{Re} s>1$. Справедливо тождество

$$
0=\left(L^{\prime}(s, X)-\frac{L^{\prime}(s, X)}{L(s, X)} L(s, X)\right) \sum_{N(C) \leqslant u} X(C) \mu_{\mathcal{K}}(C) N^{-s}(C),
$$

из которого следует, что

$$
\begin{aligned}
0=- & \sum_{N(C) \leqslant u} X(C) N^{-s}(C) \mu_{\mathcal{K}}(C) \sum_{A} X(A)(\ln N(A)) N^{-s}(A) \\
& +\sum_{B} X(B) \Lambda_{\mathcal{K}}(N(B)) N^{-s}(B) L(s, X) \sum_{N(C) \leqslant u} X(C) \mu_{\mathcal{K}}(C) N^{-s}(C) .
\end{aligned}
$$

Далее,

$$
\begin{array}{rl}
\sum_{B} & X(B) \Lambda_{\mathcal{K}}(N(B)) N^{-s}(B) L(s, X) \sum_{N(C) \leqslant u} X(C) \mu_{\mathcal{K}}(C) N^{-s}(C) \\
= & \sum_{N(C) \leqslant u} X(C) \mu_{\mathcal{K}}(C) N^{-s}(C) \sum_{N(B) \leqslant u} X(B) \Lambda_{\mathcal{K}}(N(B)) N^{-s}(B) \sum_{A} X(A) N^{-s}(A) \\
& +\sum_{N(B) \leqslant u} X(B) \Lambda_{\mathcal{K}}(N(B)) N^{-s}(B) L(s, X) \sum_{N(C) \leqslant u} X(C) \mu_{\mathcal{K}}(C) N^{-s}(C) .
\end{array}
$$


Теперь утверждение леммы следует из тождества

$$
L(s, X) \sum_{N(C) \leqslant u} X(C) \mu_{\mathcal{K}}(C) N^{-s}(C)=1+\sum_{u<N(A)} X(A) N^{-s}(A) \sum_{\substack{C \mid A \\ N(C) \leqslant u}} \mu_{\mathcal{K}}(C) .
$$

Лемма доказана.

Пусть $n \in \mathbb{N}$, и пусть

$$
\begin{gathered}
a_{X}(n)=\sum_{\substack{A \\
N(A)=n}} X(A), \quad b_{X}(n)=\sum_{\substack{A \\
N(A)=n}} X(A) \sum_{\substack{C \mid A \\
N(C) \leqslant u}} \mu_{\mathcal{K}}(C), \\
c_{X}(n)=\sum_{\substack{A \\
N(A)=n}} X(A) \mu_{\mathcal{K}}(A) .
\end{gathered}
$$

Заметим, что из известных свойств идеалов $\mathbb{Z}_{\mathcal{K}}$ (см., например, [4, с. 232]) прямо следует, что

$$
\left|a_{X}(n)\right| \ll \tau(n), \quad\left|b_{X}(n)\right| \ll \tau^{2}(n), \quad\left|c_{X}(n)\right| \ll \tau(n) .
$$

Перепишем тождество (3) в следующем виде:

$$
\begin{aligned}
\sum_{u<n} a_{X}(n) \Lambda_{\mathcal{K}}(n) n^{-s}= & \sum_{x \leqslant u} c_{X}(x) x^{-s} \sum_{l}(\ln l) a_{X}(l) l^{-s} \\
& -\sum_{x \leqslant u} c_{X}(x) x^{-s} \sum_{y \leqslant u} a_{X}(y) \Lambda_{\mathcal{K}}(y) y^{-s} \sum_{n} a_{X}(n) n^{-s} \\
& -\sum_{u<m} a_{X}(m) \Lambda_{\mathcal{K}}(m) m^{-s} \sum_{u<n} b_{X}(n) n^{-s}
\end{aligned}
$$

Лемма 7. Пусть $1 \leqslant u \leqslant N$, где $N \in \mathbb{N}$. Тогда для любой комплекснозначной функции $f(x)$ справедливо тождество

$$
\sum_{u<n \leqslant N} a_{X}(n) \Lambda_{\mathcal{K}}(n) f(n)=W_{1}-W_{2}-W_{3},
$$

¿əe

$$
\begin{gathered}
W_{1}=\sum_{x \leqslant u} c_{X}(x) \sum_{l \leqslant N x^{-1}}(\ln l) a_{X}(l) f(x l), \\
W_{2}=\sum_{x \leqslant u} c_{X}(x) \sum_{y \leqslant u} a_{X}(y) \Lambda_{\mathcal{K}}(y) \sum_{n \leqslant N(x y)^{-1}} a_{X}(n) f(x y n), \\
W_{3}=\sum_{u<m \leqslant N u^{-1}} a_{X}(m) \Lambda_{\mathcal{K}}(m) \sum_{u<n \leqslant N m^{-1}} b_{X}(n) f(m n) .
\end{gathered}
$$

ДокАзАтельство. Лемма выводится из тождества (3) тем же рассуждением, которым доказывается теорема 1 в монографии [8, с. 62].

Лемма 8. Пусть $\alpha=\frac{a}{q}+\frac{\theta}{q^{2}},(a, q)=1, q \geqslant 1,|\theta| \leqslant 1$. Тогда при любом $\beta$, $U>0, Z \geqslant 1$ имеем

$$
\sum_{x=1}^{Z} \min \left(U^{2}, \frac{1}{\|\alpha x+\beta\|^{2}}\right) \leqslant 10\left(\frac{Z}{q}+1\right) U^{2}\left(1+\frac{q}{U}\right) .
$$


ДокАЗАТЕЛЬСтво. Достаточно доказать, что при любом $\beta_{1}$

$$
\sum_{x=1}^{q} \min \left(U^{2}, \frac{1}{\left\|\alpha x+\beta_{1}\right\|^{2}}\right) \leqslant 10 U^{2}\left(1+\frac{q}{U}\right)
$$

По условию имеем

$$
\alpha x+\beta_{1}=\frac{a x+\left[\beta_{1} q\right]+\theta_{1}(x)}{q},
$$

где $\theta_{1}(x)=\frac{\theta x}{q}+\left\{\beta_{1} q\right\},\left|\theta_{1}(x)\right|<2$.

Поскольку $a$ и $q$ - взаимно простые числа, то числа $a x+\left[\beta_{1} q\right]$ пробегают вместе с $x$ полную систему вычетов по модулю $q$, следовательно,

$$
\sum_{x=1}^{q} \min \left(U^{2}, \frac{1}{\left\|\alpha x+\beta_{1}\right\|^{2}}\right) \leqslant 5 U^{2}+\sum_{3 \leqslant y \leqslant \frac{q}{2}} \min \left(U^{2}, \frac{2 q^{2}}{(y-2)^{2}}\right) .
$$

Теперь если $q \leqslant U$, то

$$
\sum_{x=1}^{q} \min \left(U^{2}, \frac{1}{\left\|\alpha x+\beta_{1}\right\|^{2}}\right) \leqslant 5 U^{2}+4 q^{2}<10 U^{2}\left(1+\frac{q}{U}\right) .
$$

Если же $U<q$, то

$$
\sum_{x=1}^{q} \min \left(U^{2}, \frac{1}{\left\|\alpha x+\beta_{1}\right\|^{2}}\right) \leqslant 5 U^{2}+U q+2 q^{2} \sum_{\frac{q}{U}<y \leqslant \frac{q}{2}} \frac{1}{(y-2)^{2}}<10 U^{2}\left(1+\frac{q}{U}\right) .
$$

Лемма доказана.

Лемма 9. Пусть $f(x)$ - действительная функиия на $[a, b], q_{1} \leqslant b-a, q_{1}-$ натуральное число. Тогда

$$
\left|\sum_{a<n \leqslant b} e^{2 \pi i f(n)}\right| \ll \frac{b-a}{\sqrt{q_{1}}}+\sqrt{\frac{b-a}{q_{1}} \sum_{r=1}^{q_{1}-1}\left|\sum_{a<n \leqslant b-r} e^{2 \pi i g(n)}\right|},
$$

где $g(n)=f(n+r)-f(n)$, а постоянная в знаке « является абсолютной.

ДокАЗАТЕЛЬСТво см. в [9, с. 26].

ЛЕмма 10. Пусть $с>0$ - произвольная постоянная, и пусть

$$
\alpha=\frac{a}{q}+\frac{\theta}{q^{2}}, \quad(a, q)=1, \quad q \geqslant 1, \quad|\theta| \leqslant 1 .
$$

Тогда существуют положительные постоянные $c_{1}$ u $c_{2}$ такие, что при $\ln ^{c_{1}} N<q \leqslant N \ln ^{-c_{2}} N$ справедлива оченка

$$
\left|S_{Q}(\alpha)\right| \ll \sqrt{N} \ln ^{-c} N
$$


ДокАЗАТЕльство. В силу следствия 2 имеем

$$
\begin{aligned}
S_{Q}(\alpha) & =\frac{1}{\alpha(Q)} \sum_{\substack{P \in \mathcal{A}^{-1} \\
N(P) \leqslant \sqrt{N}}} e^{2 \pi i \alpha N^{2}(P)}+O(\sqrt[4]{N}) \\
& =\frac{1}{\alpha(Q)} \sum_{X} \bar{X}(\mathcal{A}) \sum_{N(P) \leqslant \sqrt{N}} X(P) e^{2 \pi i \alpha N^{2}(P)}+O(\sqrt[4]{N}),
\end{aligned}
$$

где $\mathcal{A}$ - класс идеалов $\mathbb{Z}_{\mathcal{K}}$, соответствующий квадратичной форме $Q$.

Применим формулу частного суммирования и лемму 6 :

$$
\begin{aligned}
\left|S_{Q}(\alpha)\right| & \ll \sum_{X}\left|\sum_{u<n \leqslant \sqrt{N_{1}}} a_{X}(n) \Lambda_{\mathcal{K}}(n) e^{2 \pi i \alpha n^{2}}\right|+\sqrt[4]{N}+u \\
& \ll \sum_{X}\left(W_{1}(X)+W_{2}(X)+W_{3}(X)\right)+\sqrt[4]{N}+u
\end{aligned}
$$

где $N_{1}$ - то число из промежутка $(1, N]$, при котором модуль суммы максимален,

$$
\begin{aligned}
& W_{1}(X)=\sum_{x \leqslant u} \tau(x)\left|\sum_{l \leqslant \sqrt{N_{1}} x^{-1}}(\ln l) a_{X}(l) e^{2 \pi i \alpha x^{2} l^{2}}\right|, \\
& W_{2}(X)=\sum_{x \leqslant u} \tau(x) \sum_{y \leqslant u} \tau(y) \Lambda(y)\left|\sum_{n \leqslant \sqrt{N_{1}}(x y)^{-1}} a_{X}(n) e^{2 \pi i \alpha x^{2} l^{2}}\right|, \\
& W_{3}(X)=\sum_{u<m \leqslant \sqrt{N_{1}} u^{-1}} \tau(m) \Lambda(m)\left|\sum_{u<n \leqslant \sqrt{N_{1}} m^{-1}} b_{X}(n) e^{2 \pi i \alpha m^{2} n^{2}}\right| .
\end{aligned}
$$

Пусть $u=\ln ^{c_{3}} N$, где $c_{3}$ - положительная постоянная, точное значение которой мы выберем позже.

Сначала получим оценку суммы $W_{3}(X)$. Промежутки суммирования по $m$ и $n$ разобьем по геометрическим прогрессиям так, что

$$
W_{3}(X) \ll W_{3}(X, M, K) \ln ^{2} N,
$$

где

$$
W_{3}(X, M, K)=\sum_{M<m \leqslant 2 M} \tau(m) \Lambda(m)\left|\sum_{\substack{K<n \leqslant K_{1} \\ n \leqslant \sqrt{N_{1}} m^{-1}}} b_{X}(n) e^{2 \pi i \alpha m^{2} n^{2}}\right|,
$$

а $M, K, K_{1}$ - числа, удовлетворяющие условиям $u<M \leqslant \sqrt{N_{1}} u^{-1}, u<K \leqslant$ $\sqrt{N_{1}} u^{-1}, K<K_{1} \leqslant 2 K$ и такие, что сумма $W_{3}(X, M, K)$ максимальна.

Если $M K \leqslant \sqrt{N} \ln ^{-7-c} N$, то тривиальная оценка $W_{3}(X, M, K)$ приводит к неравенству

$$
W_{3}(X) \ll \sqrt{N} \ln ^{-c} N,
$$

поэтому в дальнейшем считаем, что выполняется неравенство $M K>\sqrt{N} \times$ $\ln ^{-7-c} N$. 
Применяя неравенство Коши, получаем

$$
\begin{aligned}
& \left(W_{3}(X, M, K)\right)^{2} \ll M^{2} K \ln ^{20} N \\
& \quad+M \ln ^{5} N \sum_{j=1}^{K} \sum_{K<n \leqslant K_{1}} \tau^{2}(n) \tau^{2}(n+j)\left|\sum_{\substack{M<m \leqslant 2 M \\
m \leqslant \sqrt{N_{1}}(n+j)^{-1}}} e^{2 \pi i j(2 n+j) m^{2}}\right| .
\end{aligned}
$$

Возведем обе части неравенства в квадрат и еще раз воспользуемся неравенством Коши:

$$
\begin{aligned}
\left(W_{3}(X, M, K)\right)^{4} \ll & (M K)^{4}\left(K^{-2}+M^{-1}\right) \ln ^{40} N \\
& +M^{2} K^{2} \sum_{j=1}^{K} \sum_{n \leqslant 5 K} \sum_{r \leqslant 2 M} \min \left(M, \frac{1}{\|\alpha j n r\|}\right) \ln ^{40} N \\
\ll & (M K)^{4}\left(K^{-2}+M^{-1}\right) \ln ^{40} N \\
& +M^{2} K^{2} \sum_{n \leqslant 10 M K^{2}} \tau_{3}(n) \min \left(M, \frac{1}{\|\alpha n\|}\right) \ln ^{40} N .
\end{aligned}
$$

Далее,

$$
\left(\sum_{n \leqslant 10 M K^{2}} \tau_{3}(n) \min \left(M, \frac{1}{\|\alpha n\|}\right)\right)^{2} \ll V \sum_{n \leqslant 10 M K^{2}} \tau_{3}^{2}(n),
$$

где $V=\sum_{n \leqslant 10 M K^{2}} \min \left(M^{2}, \frac{1}{\|\alpha n\|^{2}}\right)$. В силу леммы 8 имеем

$$
W_{3}(X, M, K) \ll M K\left(\frac{1}{K^{2}}+\frac{1}{\sqrt{M}}+\frac{1}{\sqrt{q}}+\frac{\sqrt{q}}{M K}\right) \ln ^{11} N .
$$

Отсюда и из неравенств $\ln ^{c_{1}} N<q \leqslant N \ln ^{-c_{2}} N, K>u=\ln ^{c_{3}} N, \quad M>u=$ $\ln ^{c_{3}} N, \sqrt{N} \ln ^{-7-c}<M K \leqslant \sqrt{N}$ следует, что

$$
W_{3}(X, M, K) \ll \sqrt{N}\left((\ln N)^{\frac{22-c_{1}}{2}}+(\ln N)^{11-\frac{c_{3}}{2}}+(\ln N)^{\frac{36+2 c-c_{2}}{2}}\right) .
$$

Пусть выполняются неравенства

$$
c_{1} \geqslant 2 c+26, \quad c_{2} \geqslant 4 c+40, \quad c_{3} \geqslant 2 c+26 .
$$

Тогда справедливы оценки

$$
W_{3}(X, M, K) \ll \sqrt{N} \ln ^{-c-2} N, \quad W_{3}(X) \ll \sqrt{N} \ln ^{-c} N .
$$

Переходим к оценке сумм $W_{1}(X)$ и $W_{2}(X)$. Они оцениваются одинаково. Оценим $W_{2}(X)$.

Рассмотрим внутреннюю сумму

$$
S_{2}(\beta)=\sum_{n \leqslant N_{2}} a_{X}(n) e^{2 \pi i \beta n^{2}}=\sum_{N(A) \leqslant N_{2}} X(A) e^{2 \pi i \beta N^{2}(A)},
$$

где $N_{2}=\sqrt{N_{1}}(x y)^{-1}, 1 \leqslant x, y \leqslant u, \beta=\alpha x^{2} y^{2}$. 


\section{Имеем}

$$
S_{2}(\beta)=\sum_{\mathcal{B}} X(\mathcal{B}) \sum_{\substack{A \in \mathcal{B} \\ N(A) \leqslant N_{2}}} e^{2 \pi i \beta N^{2}(A)}=\sum_{\mathcal{B}} X(\mathcal{B}) \sum_{\substack{t, v \\ Q_{\mathcal{B}}(t, v) \leqslant N_{2}}} e^{2 \pi i \beta Q_{\mathcal{B}}^{2}(t, v)},
$$

где $Q_{\mathcal{B}}(t, v)$ - квадратичная форма, отвечающая классу идеалов $\mathcal{B}$ (см. [3, с.215, теорема 154]).

Разобьем промежутки суммирования по $t$ и $v$ на интервалы $T<t \leqslant 2 T$ и $V<v \leqslant 2 V$. Тогда

$$
\left|\sum_{\substack{t, v \\ Q_{\mathcal{B}}(t, v) \leqslant N_{2}}} e^{2 \pi i \beta Q_{\mathcal{B}}^{2}(t, v)}\right| \ll\left|\sum_{\substack{T<t \leqslant 2 T \\ V<v \leqslant 2 V \\ Q_{\mathcal{B}}(t, v) \leqslant N_{2}}} e^{2 \pi i \beta Q_{\mathcal{B}}^{2}(t, v)}\right| \ln ^{2} N,
$$

где $T$ и $V$ - такие числа, что $T \ll \sqrt{N_{2}}, V \ll \sqrt{N_{2}}$, а модуль суммы в правой части (7) максимален.

Пусть $T \geqslant V$, тогда

$$
\left|\sum_{\substack{T<t \leqslant 2 T \\ V<v \leqslant 2 V \\ Q_{\mathcal{B}}(t, v) \leqslant N_{2}}} e^{2 \pi i \beta Q_{\mathcal{B}}^{2}(t, v)}\right| \leqslant \sum_{V<v \leqslant 2 V}\left|\sum_{\substack{T<t \leqslant 2 T \\ Q_{\mathcal{B}}(t, v) \leqslant N_{2}}} e^{2 \pi i \beta Q_{\mathcal{B}}^{2}(t, v)}\right| .
$$

Если промежуток изменения $t$, заданный неравенствами $T<t \leqslant 2 T$, $Q_{\mathcal{B}}(t, v) \leqslant N_{2}$, имеет длину, не превосходящую $\sqrt[4]{N} u^{-2} \ln ^{-5-c} N$, то тривиально получаем, что

$$
\left|W_{2}(X)\right| \ll \sqrt{N} \ln ^{-c} N .
$$

Поэтому в дальнейшем считаем, что промежуток изменения $t$, который мы обозначим через $\left(t_{1}, t_{2}\right]$, имеет длину, бо́льшую, чем $\sqrt[4]{N} u^{-2} \ln ^{-5-c} N$.

К сумме

$$
S_{3}(\beta)=\sum_{t_{1}<t \leqslant t_{2}} e^{2 \pi i \beta Q_{\mathcal{B}}^{2}(t, v)}
$$

применим лемму 9:

$$
\left|S_{3}(\beta)\right|^{2} \ll \frac{\left(t_{2}-t_{1}\right)^{2}}{q_{1}}+\frac{t_{2}-t_{1}}{q_{1}} \sum_{r_{1}=1}^{q_{1}-1}\left|\sum_{t_{1}<t \leqslant t_{2}-r_{1}} e^{2 \pi i \beta g_{1}(t)}\right|,
$$

где $g_{1}(t)$ - многочлен степени 3 от $t$ с коэффициентами, зависящими от $v$, со старшим коэффициентом $4 a^{2} r_{1}, q_{1}=\left(t_{2}-t_{1}\right) / 10, a-$ коэффициент при $t^{2}$ формы $Q_{\mathcal{B}}(t, v)$ (здесь $a \neq 0$, так как квадратичная форма $Q_{\mathcal{B}}(t, v)$ положительно определена).

Возведем обе части (8) в квадрат, пользуясь неравенством Коши:

$$
\left|S_{3}(\beta)\right|^{4} \ll \frac{\left(t_{2}-t_{1}\right)^{4}}{q_{1}^{2}}+\frac{\left(t_{2}-t_{1}\right)^{2}}{q_{1}} \sum_{r_{1}=1}^{q_{1}-1}\left|\sum_{t_{1}<t \leqslant t_{2}-r_{1}} e^{2 \pi i \beta g_{1}(t)}\right|^{2},
$$

и применим лемму 9:

$$
\left|S_{3}(\beta)\right|^{4} \ll \frac{\left(t_{2}-t_{1}\right)^{4}}{q_{1}}+\frac{\left(t_{2}-t_{1}\right)^{3}}{q_{1}^{2}} \sum_{r_{1}=1}^{q_{1}-1} \sum_{r_{2}=1}^{q_{1}-1}\left|\sum_{t_{1}<t \leqslant t_{2}-r_{1}-r_{2}} e^{2 \pi i \beta g_{2}(t)}\right|,
$$


где $g_{2}(t)$ - многочлен степени 2 от переменной $t$ со старшим коэффициентом $12 a^{2} r_{1} r_{2}$.

Вновь возводя обе части полученного неравенства в квадрат и пользуясь леммой 9 , имеем

$$
\left|S_{3}(\beta)\right|^{8} \ll \frac{\left(t_{2}-t_{1}\right)^{8}}{q_{1}}+\frac{\left(t_{2}-t_{1}\right)^{7}}{q_{1}^{3}} \sum_{r_{1}=1}^{q_{1}-1} \sum_{r_{2}=1}^{q_{1}-1} \sum_{r_{3}=1}^{q_{1}-1}\left|\sum_{t_{1}<t \leqslant t_{2}-r_{1}-r_{2}-r_{3}} e^{2 \pi i \beta 24 a^{2} r_{1} r_{2} r_{3} t}\right| .
$$

Отсюда и из леммы 8 получаем

$$
\left|S_{3}(\beta)\right|^{8} \ll \frac{\left(t_{2}-t_{1}\right)^{8}}{q_{1}}+\frac{\left(t_{2}-t_{1}\right)^{7}}{q_{1}^{3}} \sum_{n \leqslant 24 a^{2} u^{2} q_{1}^{3}} \tau_{4}(n) \min \left(t_{2}-t_{1}, \frac{1}{\|\alpha n\|}\right) .
$$

Далее, из известного неравенства $\sum_{n \leqslant K} \tau_{4}^{2}(n) \ll K \ln ^{15} K$ (см. [9, с. 59]) и леммы 8 получаем

$$
\begin{aligned}
\left|S_{3}(\beta)\right|^{8} & \ll \frac{\left(t_{2}-t_{1}\right)^{8}}{q_{1}} \\
& +\frac{\left(t_{2}-t_{1}\right)^{7}}{q_{1}^{3}} \sqrt{\sum_{n \leqslant 24 a^{2} u^{2} q_{1}^{3}} \tau_{4}^{2}(n) \sum_{n \leqslant 24 a^{2} u^{2} q_{1}^{3}} \min \left(\left(t_{2}-t_{1}\right)^{2}, \frac{1}{\|\alpha n\|^{2}}\right)} \\
& \ll\left(t_{2}-t_{1}\right)^{8}\left(\frac{u^{2}}{\sqrt{q}}+\frac{u^{2}}{\sqrt{q_{1}}}+\frac{u \sqrt{q}}{q_{1}^{2}}\right) \ln ^{8} N .
\end{aligned}
$$

Параметры определены так, что

$$
\ln ^{c_{1}} N<q \leqslant N \ln ^{-c_{2}} N, \quad \sqrt[4]{N} u^{-2} \ln ^{-5-c} N<q_{1} \leqslant \sqrt[4]{N}(x y)^{-1},
$$

откуда имеем оценки

$$
\begin{gathered}
\left|S_{3}(\beta)\right| \ll \sqrt[4]{N}(x y)^{-1}\left((\ln N)^{\frac{16+4 c_{3}-c_{1}}{16}}+(\ln N)^{\frac{36+10 c_{3}+4 c-c_{2}}{16}}\right), \\
\left|S_{2}(\beta)\right| \ll \sqrt[4]{N}(x y)^{-1}\left((\ln N)^{\frac{48+4 c_{3}-c_{1}}{16}}+(\ln N)^{\frac{68+10 c_{3}+4 c-c_{2}}{16}}\right), \\
\left|W_{2}(X)\right| \ll \sqrt{N}\left((\ln N)^{\frac{80+4 c_{3}-c_{1}}{16}}+(\ln N)^{\frac{100+10 c_{3}+4 c-c_{2}}{16}}\right) .
\end{gathered}
$$

Выберем $c_{3}=2 c+26$. Пусть

$$
c_{1} \geqslant 184+24 c, \quad c_{2} \geqslant 360+40 c .
$$

Тогда имеем

$$
\left|W_{2}(X)\right| \ll \sqrt{N} \ln ^{-c} N .
$$

Кроме того, из (9) следует, что справедливы неравенства (5), а следовательно, и (6). Лемма доказана.

\section{§ 4. Доказательство теоремы 1}

1. Пусть $c_{1}$ и $c_{2}$ удовлетворяют неравенствам (9). Представим $I(N)$ в виде интеграла

$$
I(N)=\int_{-\frac{1}{\tau}}^{1-\frac{1}{\tau}} S_{Q_{1}}(\alpha) \cdots S_{Q_{5}}(\alpha) e^{-2 \pi i \alpha N} d \alpha
$$

где $\tau=N \ln ^{-c_{2}} N$. 
Определим множества $E_{1}$ и $E_{2}$ :

$$
\begin{aligned}
E_{1}=\left\{\alpha \in\left(-\frac{1}{\tau}, 1-\frac{1}{\tau}\right], \alpha\right. & \left.=\frac{a}{q}+z,(a, q)=1,1 \leqslant q \leqslant \ln ^{c_{1}} N,|z| \leqslant \frac{1}{q \tau}\right\}, \\
E_{2} & =\left(-\frac{1}{\tau}, 1-\frac{1}{\tau}\right] \backslash E_{1} .
\end{aligned}
$$

Тогда

$$
\begin{aligned}
I(N)= & \int_{E_{1}} S_{Q_{1}}(\alpha) \cdots S_{Q_{5}}(\alpha) e^{2 \pi i \alpha N} d \alpha \\
& +\int_{E_{2}} S_{Q_{1}}(\alpha) \cdots S_{Q_{5}}(\alpha) e^{2 \pi i \alpha N} d \alpha=I_{1}(N)+I_{2}(N) .
\end{aligned}
$$

2. Вычислим $I_{1}(N)$. Пусть $Q$ - одна из квадратичных форм $Q_{1}, \ldots, Q_{5}, h-$ число классов эквивалентных форм того же дискриминанта, что и $Q$, а $\chi-$ один из характеров $\chi_{1}, \ldots, \chi_{5}$. Сначала докажем, что

$$
S_{Q}\left(\frac{a}{q}+z\right)=\frac{S^{\prime}(a, q)+\chi(q ; D, 0) S^{\prime \prime}(a, q)}{\varphi(q) h \alpha(Q)} J(z)+O\left(\sqrt{N} \exp \left\{-\frac{c_{0}}{3}(\ln N)^{\gamma}\right\}\right),
$$

где $c_{0}>0-$ константа из леммы $5, \gamma=\frac{1}{20 c_{1}}$. Преобразуем $S_{Q}\left(\frac{a}{q}+z\right)$ :

$$
\begin{aligned}
S_{Q}\left(\frac{a}{q}+z\right) & =\sum_{\sqrt[4]{N}<p \leqslant \sqrt{N}} \exp \left\{2 \pi i\left(\frac{a}{q}+z\right) p^{2}\right\}+O(\sqrt[4]{N}) \\
& =\sum_{l=1}^{*} \exp \left\{2 \pi i \frac{a l^{2}}{q}\right\} T(l)+O(\sqrt[4]{N})
\end{aligned}
$$

где

$$
T(l)=\sum_{\substack{4 \\ \sqrt[4]{N}<p \leqslant \sqrt{N} \\ p \equiv l(\bmod q)}}^{\prime} e^{2 \pi i z p^{2}}
$$

Применим формулу частного суммирования:

$$
\begin{aligned}
T(l)= & \sum_{\sqrt[4]{N}<n \leqslant \sqrt{N}} e^{2 \pi i z n^{2}}\left(\pi_{Q}(n, q, l)-\pi_{Q}(n-1, q, l)\right) \\
=- & \int_{\sqrt[4]{N}}^{\sqrt{N}}\left(\pi_{Q}(x, q, l)-\pi_{Q}(\sqrt[4]{N}, q, l)\right) d e^{2 \pi i z x^{2}} \\
& +\left(\pi_{Q}(\sqrt{N}, q, l)-\pi_{Q}(\sqrt[4]{N}, q, l)\right) e^{2 \pi i z N} .
\end{aligned}
$$

Воспользуемся следствием 2 и леммой 5 и проинтегрируем по частям:

$$
T(l)=\frac{1+\chi(q ; D, 0) \chi(l)}{\varphi(q) h \alpha(Q)} J(z)+O\left(\sqrt{N} \exp \left\{-\frac{c_{0}}{2}(\ln N)^{\gamma}\right\}\right) .
$$

Подставим эту формулу в (11) и получим (10). 

чаем

Далее, применяя неравенство $J(z) \ll \frac{1}{\sqrt{|z|}}$ (см., например, [10, с. 30]), полу$\int_{-\frac{1}{q \tau}}^{\frac{1}{q \tau}} J^{5}(z) e^{-2 \pi i z N} d z=H(N)+O\left((q \tau)^{\frac{3}{2}}\right)=H(N)+O\left(N^{\frac{3}{2}}(\ln N)^{\frac{3}{2}\left(c_{1}-c_{2}\right)}\right)$.

Из последнего равенства следует, что

$$
\begin{aligned}
I_{1}(N)= & \frac{1}{h_{1} \ldots h_{5} \alpha\left(Q_{1}\right) \ldots \alpha\left(Q_{5}\right)} \\
& \times \sum_{q \leqslant \ln ^{c_{1}} N} \gamma\left(q, D_{1}, \ldots, D_{5}, N\right)\left(H(N)+O\left(N^{\frac{3}{2}}(\ln N)^{\frac{3}{2}\left(c_{1}-c_{2}\right)}\right)\right) .
\end{aligned}
$$

Теперь пользуясь леммой 4, получаем равенство

$$
\begin{aligned}
I_{1}(N)= & \frac{1}{h_{1} \ldots h_{5} \alpha\left(Q_{1}\right) \ldots \alpha\left(Q_{5}\right)} \sigma\left(N, D_{1}, \ldots, D_{5}\right) H(N) \\
& +O\left(N^{\frac{3}{2}}(\ln N)^{\frac{3}{2}\left(c_{1}-c_{2}\right)}\right)+O\left(N^{\frac{3}{2}}(\ln N)^{-\frac{1}{2} c_{1}+5 \varepsilon}\right) .
\end{aligned}
$$

3. Оценим $I_{2}(N)$. Пользуясь леммой 10 и неравенством Коши, имеем

$$
\left|I_{2}(N)\right| \leqslant \max _{\alpha \in E_{2}}\left|S_{Q_{5}}(\alpha)\right| \prod_{j=1}^{4}\left(\int_{0}^{1}\left|S_{Q_{j}}(\alpha)\right|^{4} d \alpha\right)^{\frac{1}{4}} \ll \sqrt{N} \sum_{n \leqslant N} r^{2}(n) \ln ^{-c} N,
$$

где $r(n)$ - количество представлений числа $n$ суммой двух квадратов.

Поскольку $\sum_{n \leqslant N} r^{2}(n) \ll \sum_{n \leqslant N} \tau^{2}(n) \ll N \ln ^{3} N$, получено неравенство

$$
I_{2}(N) \ll N^{\frac{3}{2}} \ln ^{3-c} N
$$

Пусть $c=b+3, c_{1}=24 b+256, c_{2}=40 b+480$. Теперь утверждение теоремы следует из (12) и (13).

Асимптотическая формула для $H(N)$ выведена в монографии [10, с. 142].

СледСтвиЕ 3. Пусть выполнены условия теоремы 1 и модули дискриминантов квадратичных форм $D_{1}, \ldots, D_{5}$ больше некоторой абсолютной постоянной $D_{0}$. Тогда существует абсолютная постоянная $\Delta>0$ такая, что

$$
\gamma\left(q, N, D_{1}, \ldots, D_{5}\right)>\Delta .
$$

ДокАЗАтЕльство. Раскроем скобки в произведении

$$
\prod_{j=1}^{5}\left(\frac{S^{\prime}(a, q)}{\varphi(q)}+\frac{\chi\left(q ; D_{j}, 0\right) S_{j}^{\prime \prime}(a, q)}{\varphi(q)}\right) \exp \left\{-2 \pi i \frac{a}{q} N\right\}
$$

получим тридцать два слагаемых, которым соответствует тридцать два сингулярных ряда.

Один из этих рядов является сингулярным рядом классической задачи Хуа Ло-кена. В монографии [1] эта сумма оценена снизу абсолютной постоянной, которую мы обозначим через $2 \Delta$. 
Суммы остальных рядов оценим сверху. Для них рассуждения аналогичны, поэтому ограничимся верхней оценкой суммы ряда

$$
\sigma_{5}\left(N, D_{5}\right)=\sum_{q=1}^{\infty} \sum_{a=0}^{q-1}\left(\frac{S^{\prime}(a, q)}{\varphi(q)}\right)^{4} \chi\left(q ; D_{5}, 0\right) \frac{S_{5}^{\prime \prime}(a, q)}{\varphi(q)} \exp \left\{-2 \pi i \frac{a}{q} N\right\} .
$$

Имеем

$$
\sigma_{5}\left(N, D_{5}\right) \ll \sum_{k=1}^{\infty} \sum_{a=0}^{D_{5} k-1} \frac{\left|S^{\prime}\left(a, D_{5} k\right)\right|^{4}\left|S_{5}^{\prime \prime}\left(a, D_{5} k\right)\right|}{\varphi^{5}\left(D_{5} k\right)} .
$$

Пользуясь леммой 4 , получаем

$$
\sigma_{5}\left(N, D_{5}\right) \ll \frac{D_{5}^{\frac{5}{2}+\varepsilon}}{\varphi^{4}\left(D_{5}\right)} .
$$

Однако по условию $D_{5} \geqslant D_{0}$. Параметр $D_{0}$ выбираем столь большим, чтобы суммы тридцати одного сингулярного ряда не превосходили по модулю $\Delta$.

\section{Список литературы}

1. Ло-кен Хуа, Аддитивная теория простых чисел, Тр. МИАН, 22, 1947; англ. пер.: Loo-keng Hua, Additive theory of prime numbers, Transl. Math. Monogr., 13, Amer. Math. Soc., Providence, RI, 1965.

2. З. И. Боревич, И. Р. Шафаревич, Теория чисел, Наука, М., 1985; англ. пер. 1-го изд.: Z. I. Borevich, I. R. Shafarevich, Number theory, Pure Appl. Math., 20, Academic Press, New York-London, 1966.

3. Э. Гекке, Лекиии по теории алгебраических чисел, ГИТТЛ, М.-Л., 1940; пер. с нем.: E. Hecke, Vorlesungen über die Theorie der algebraischen Zahlen, Akad. Verlagsges, Leipzig, 1923.

4. К.Ф. Айерленд, М.И. Роузен, Классическое введение в современную теорию чисел, Мир, М., 1987; пер. с англ.: К. F. Ireland, M. I. Rosen, A classical introduction to modern number theory, Grad. Texts in Math., 84, Springer-Verlag, New YorkHeidelberg-Berlin, 1982.

5. С.А. Гриценко, "О функциональном уравнении одного арифметического ряда Дирихле", Тр. V Международной конференции "Алгебра и теория чисел: современные проблемы и приложения" (Россия, Тула, 2003), Чебышевский сб., 4:2(6) (2003), 55-67.

6. С.А. Гриценко, "О распределении норм простых идеалов из заданного класса в арифметических прогрессиях", Записки науч. сем. ПОМИ, 322 (2005), 45-62.

7. Loo Keng Hua, Introduction to number theory, Springer-Verlag, Berlin-HeidelbergNew York, 1982.

8. С. М. Воронин, А.А. Карацуба, Дзета-функция Римана, Физматлит, М., 1994; S. M. Voronin, A. A. Karatsuba, The Riemann zeta-function, de Gruyter Exp. Math., 5, Walter de Gruyter and Co., Berlin, 1992.

9. А. А. Карацуба, Основы аналитической теории чисел, Наука, М., 1983; англ. пер.: А. A. Karatsuba, Basic analytic number theory, Springer-Verlag, Berlin, 1993.

10. И. М. Виноградов, Метод тригонометрических сумм в теории чисел, Наука, М., 1980; I. M. Vinogradov, The method of trigonometrical sums in the theory of numbers, Dover Publ., Inc., Mineola, NY, 2004.

C. А. Гриценко (S. A. Gritsenko)

Белгородский государственный университет

Поступило в редакцию

E-mail: gritsenko@bsu.edu.ru 10.05.2006 